\title{
THE PHOTOGRAPHIC OBSERVATIONS OF SOME PLANETARY NEBULAE
}

\author{
XIANGLIANG HAO \\ Beijing Astronomical Observatory, Beijing, China
}

The first planetary nebula was discovered by Messier in 1794 . But for some reasons it has not been studied detail for a long time, especially for the central star Of planetary nebula. The primary research for these objects showed that the lifetime of a planetary nebula is about $510^{4}$ years, but in this period the luminosity of central star varies from $63 L_{\odot}$ to nearly $3.510^{4} L_{\odot}$ and then decrease to $100 L_{\odot}$;its temperature changes from $3.410^{4}$ to $10^{5} \mathrm{~K}$ and then begins to decrease (Seaton 1966). The radius of central stars also have fast varies in planetary nebula phase. For these reasons we consider that in the planetary nebula phase the activities of central star is very drastic and the result of these activities must cause some variation at the surface of central star witch may be detected on the earth, especially for the surface light variations. Some observers have been trying to find the luminosity variations in central stars. But until now no one has made systematical survey for these. Since the different authors used different instruments amd different processing methods at different places which may be caused a lot of uncertainty in the photometry of planetary nebulae and central stars. So it is hard to decide whether the differences between the authors or the essential variations of the objects is responsible of the observing differences. Therefore, we have selected over fifty planetary nebulae to observe for a long period at Beijing Observatory using the same instrument and the same processing method. From these observations we may determine the light variations and the brightness of the planetary nebulae and central stars more correctly.

Up to now we have taken over thousand photographic plates of planetary nebulae using the $40 / 200 \mathrm{~cm}$ double astrograph and others at Beijing Observatory since 1979. Some of these observations we have made processing and published in other papers.

\section{References}

M.J. Seaton, 1966, Mon. Not. Roy. Astron. Soc. 132, 113 